\title{
Convolution Integral: How a Graphical Type of Solution Can Help Minimize Misconceptions
}

\author{
Hendra J. Tarigan ${ }^{1 *}$ \\ ${ }^{1}$ Study Program of Electrical Engineering, President University, Bekasi 17550, Indonesia \\ *Corresponding author: tarigan@ president.ac.id
}

\begin{abstract}
A physical system, Low Pass Filter (LPF) RC Circuit, which serves as an impulse response and a square wave input signal are utilized to derive the continuous time convolution (convolution integrals). How to set up the limits of integration correctly and how the excitation source convolves with the impulse response are explained using a graphical type of solution. This in turn, help minimize the students' misconceptions about the convolution integral. Further, the effect of varying the circuit elements on the shape of the convolution output plot is presented allowing students to see the connection between a convolution integral and a physical system. PSpice simulation and experiment results are incorporated and are compared with those of the analytical solution associated with the convolution integral.
\end{abstract}

Keywords: Analytical, Convolution, Graphical, Impulse Response

\section{INTRODUCTION}

A convolution integral for a continuous time signal is part of a Signals and Systems course, which is taught to Electrical Engineering (EE) major and other engineering disciplines including Aeronautics and Astronautics. Inherent difficulties that many engineering students face when solving a convolution integral is evident. The authors of a Signals and Systems textbook have pointed out that according to an article that appeared in the IEEE Spectrum (March 1991, p.60), convolution has driven many EE students to "Contemplate theology either for salvation or as an alternative career," [1]. Analytical solutions to a convolution integral have been presented elsewhere [2-3]. Goldberg et al. has presented a systematic analytical method of evaluating a convolution integral using a superposition technique [2]. They claim that students find the semi graphical methods of solving the convolution integral difficult to follow and are susceptible to errors, in particular, when it comes to dealing with discontinuous functions. Soares et al. also presented their work on the solution to a convolution integral, which is based on an analytical solution. The technique they presented in this work can be found in any Signals and Systems textbook [3].

Students who took Signals and Systems have been interviewed by two groups of researchers at the Massachusetts Institute of Technology (MIT) and George Mason University, respectively [4-5]. Nasr et al. carried out studies on the student misconceptions and their origins in Signals and System course at MIT [4]. For a nongraphical convolution integral type of solution, they discovered that students had problems setting the integral limits. Although the students knew how to write the convolution integral equation, they had a poor contextual grasp of the integral equation itself. They had difficulties understanding the expression for the impulse response $\mathrm{h}(\mathrm{t}-$ $\tau)$. For a graphical type of solution, the students did not understand that the variable $t$ in the excitation function $x(t)$ is not the same as that in the impulse response $\mathrm{h}(\mathrm{t})$. The authors pointed out that the variable $t$ in the excitation function $\mathrm{x}(\mathrm{t})$ corresponds to a "clock" time when the input starts to occur, while that in the impulse response signifies a relative time, which is the length of time since the impulse response happens at the excitation signal. They pointed out further that good mapping between the physics and mathematics is required allowing students to build good physical intuition. In turn, this equips the students well when it comes to studying a concept heavy subject such as Signals and Systems.

In a closely related topic in Signals and Systems, Nelson et al. studied the students' understanding of convolution sum (for discrete time signals) at George Mason University, Fairfax, VA, USA [5]. They discovered that one particular group of students attempted to use the brute-force solution strategy while working on the convolution sum resulting in an incorrect solution. They discovered that there is a need for students to think conceptually rather than just applying equations or procedures. Their findings show that students in this study are challenged by their ability to utilize graphs and employ them in a correct manner.

Tagneli et al. presented an effective strategy on how a Signal and Systems course should be run [6]. Such a strategy involves peer facilitated tutorials, optional class test, in class lab assessments and the incorporating of 
interactive animations. They acknowledge the fact that Signals and Systems is a concept heavy subject where the students are required to be able to visualize signal operations including convolution. They mentioned that good animations that are capable of showing signal operations are hard to find. They mentioned further that the best animation tool available is the Educational MATLAB GUI from Georgia Tech, which provides a toolbox in order to engage MATLAB interactive animations. (GUI is short for Graphical User Interface). This group pointed out that the Educational MATLAB GUI cconvdemo for continuous time signals does not allow for custom waveforms to be used. However, the discrete time convolution animation dconvdemo allows. They realize the fact that students need to see how convolution works for a variety of input waveforms.

It has been claimed that students perceive the semi graphical type of solution to be susceptible to errors, especially when piecewise discontinue signals are involved [2]. In the present work a square wave input, which is a piecewise discontinuous signal is employed and the presented analytical solution works well with such a discontinuous signal.

The perception that many EE students have on the fact that convolution is a difficult concept to grasp [1], the misconceptions that engineering students have when it comes to dealing with a continuous time convolution as a consequence of lacking of physical intuition [4], and the students' inability to incorporate graphical type of solution to the convolution problem in general [4,5] are the motivating factors of the present paper. Presenting a graphical type of solution to the convolution integral and showing a connection between a physical system (the LPF $\mathrm{RC}$ circuit) and a convolution are the prime objectives of the present paper, which in turn, help minimize misconceptions that students have about the convolution itself. Incorporating the experiment and PSpice simulation results has its own merit. PSpice can verify fast whether or not the students' analytical solution is correct. It is hoped that the experiment results would convince the students in the way that convolution can be applied in a physical system. Further, PSpice simulation comes handy because students can change the values of $\mathrm{R}, \mathrm{C}$, frequency and the types of input (square-, triangular- or sinusoidal-wave) with a minimum effort and attain the convolution output fairly quickly.

\section{The ANALYTICAL SOLUTION}

Eqs. (1-2) represent the convolution integrals for a continuous time signal [7-8]

$$
\begin{aligned}
& y(t)=\int_{0}^{t} h(\tau) x(t-\tau) d \tau, \\
& y(t)=\int_{0}^{t} x(\tau) h(t-\tau) d t,
\end{aligned}
$$

where $\mathrm{h}(\mathrm{t})$ and $\mathrm{x}(\mathrm{t})$ are the impulse response and input signal, respectively. Fig. 1 depicts an LPF type of an RC ciruit, which will be used as a physical model for an impulse response in the convolution integral through out this paper. The s-domain transfer function $\mathrm{H}(\mathrm{s})$, cutoff frequency, inverse Laplcae Transform of $\mathrm{H}(\mathrm{s})$, and the impulse response in the time-domain are represented by Eqs. (3-6), respectively. As shown in Eqs. (5-6), the the inverse Laplace Transform of the transfers function in the s-domain gives a time-domain impulse response $\mathrm{h}(\mathrm{t})$ associated with the LPF/RC circuit depicted in in Fig. 1. The dependence of the convolution integral on the cutoof frequency $\omega_{\mathrm{c}}$, therefore, on the circuit passive elements (R and C) is shown by Eq. (7).

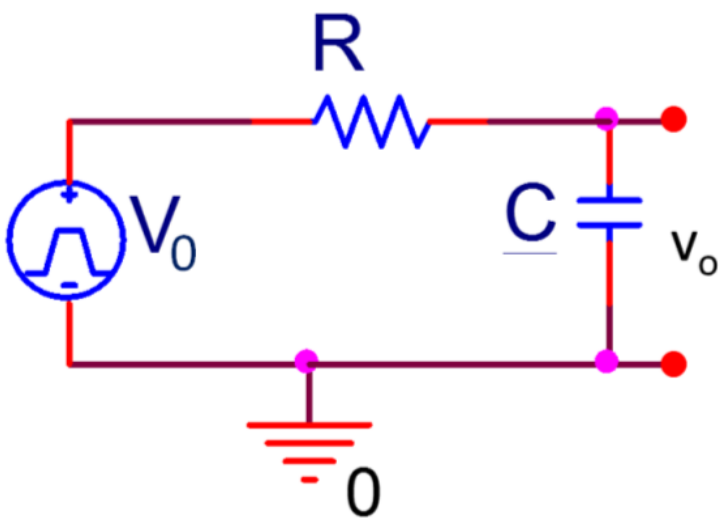

Figure 1. An RC Circuit of An LPF Type

$$
\begin{gathered}
H(s)=\frac{\omega_{c}}{s+\omega_{c}} \\
\omega_{c}=\frac{1}{R C} \\
\mathcal{L}^{-1}\{H(s)\}=\omega_{c} \exp \left(-\omega_{c} t\right) \\
h(t)=\omega_{c} \exp \left(-\omega_{c} t\right) \\
v_{o}(t)=\int_{t_{0}}^{t}\left[\omega_{c} \exp \left(-\omega_{c} \tau\right)\right] v(t-\tau) d \tau
\end{gathered}
$$

The continuous-time convolution integral shown in Eq. (1) will be used in this paper. Eqs. (8-11) represent the convolution integrals associated with the square wave input signal. Each of these equations is evaluated at a different time interval. The respective integrals are easy to evaluate. The answers are not included in this paper due to a large space that they require.

The graphical representation of the impulse response $\mathrm{h}(\mathrm{t})$ is depicted in Fig. (2), which was plotted based on Eq. (6), where $\mathrm{R}=2.2 \mathrm{k} \Omega$ and $\mathrm{C}=1.026 \mu \mathrm{F}$. In this paper, a strong emphasis is placed on the graphical type of solution in the derivation of the convolution integrals.

\section{A. For $0<t<0.5 T$}

In the discussions to follow the period of the input signal, denoted T, is $33 \mathrm{~ms}$. Depicted in Fig. (3) is a plot of $\mathrm{v}(\mathrm{t}-\tau)$ as a function of $\tau$. The blue exponentially decaying plot (with a shaded region underneath it) is the impulse response $h(\tau)$. Fig. 3(a) represents $t=0$, where the input signal is located at $\tau=0^{-}$. As we are dealing with a Linear Time Invariant (LTI) system, causality dictates that $h(\tau)$ is 0 for $\tau<0$, therefore, the convolution integral gives a zero value under this condition.

In Fig. 3(b) the leading edge of the input signal $v(t-\tau)$ 
is located at $\mathrm{t}$ (red online). This is a snapshot of the convolution. We wish to evaluate the convolution integral within the interval $0<\mathrm{t}<0.5 \mathrm{~T}$, therefore, we must set the upper limit of the integral to t. Remember that for every single value of $t$, the integral in Eq. (1) is evaluated from the lower limit $(0)$ to the upper limit $(t)$, where the variable of the integrand is $\tau$, with $t$ being a fixed value. At the instant shown in Fig. 3(b) the value of the convolution integral is the product of the area formed by the impulse response plot and the $\tau$-axis (excluding the part where the input signal is zero) and that of the corresponding overlapping area for $0<\tau<\mathrm{t}$. We shall call this product of areas PROD. The expression for the convolution integral is given by Eq. (8), where $\mathrm{V}_{0}$ signifies the amplitude of the input signal.

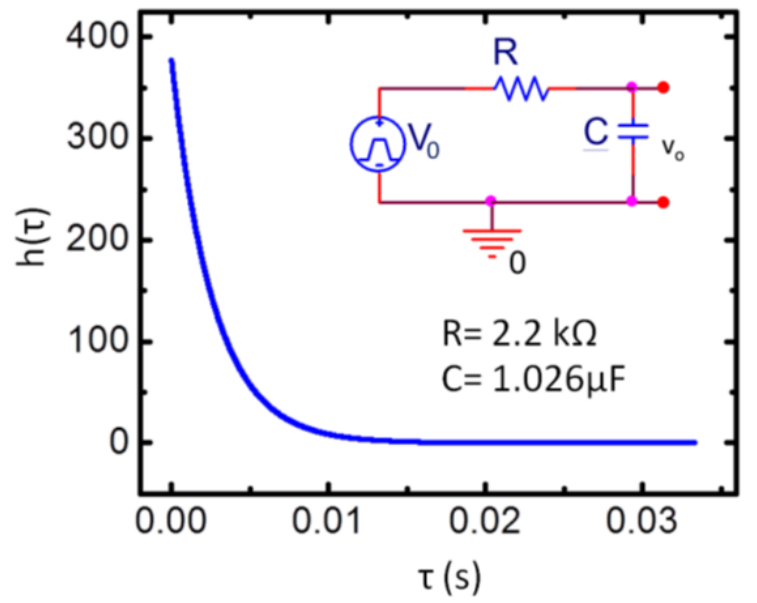

Figure 2. The Impulse Response $h(\tau)$ as a Function of $\tau$ Based on the LPF Type of an RC Circuit and Eq. (6). $R=2.2 \mathrm{k} \Omega$ and $\mathrm{C}=1.026$ $\mu \mathbf{F}$

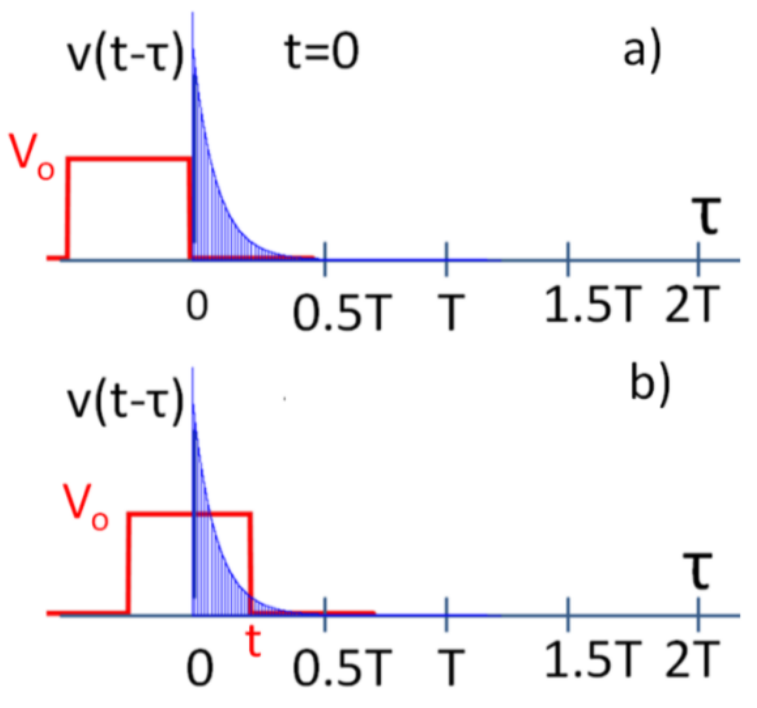

Figure 3. v(t- $\tau)$ vs $\tau$ for: a) $t=0$; and b) $0<t<0.5 T$

$$
v_{o}(t)=\int_{0}^{t} V_{0} \omega_{c} \exp \left(-\omega_{c} \tau\right) d \tau
$$

\section{B. For $0.5 T<t<T$}

As $t$ increases further, we look at another snapshot of the convolution in Fig. 4(b) where the leading edge of the input signal is located at $\mathrm{t}$. We are interested in the value of the integral within the interval $0.5 \mathrm{~T}<\mathrm{t}<\mathrm{T}$. Similar to the previous case, the upper and lower limits are $t$ and $t-0.5 \mathrm{~T}$ (at the tips of the red arrows (on line)). The convolution integral is represented by Eq. (9). At the instant shown in Fig. 4(a), where $t=0.5 \mathrm{~T}$ the value of the integral is the largest as indicated by the largest overlapping area between the input signal and the impulse response. In contrast, at the instant shown in Fig. 4(b) the overlapping area is very small leading to a small value of PROD, i.e., the integral.

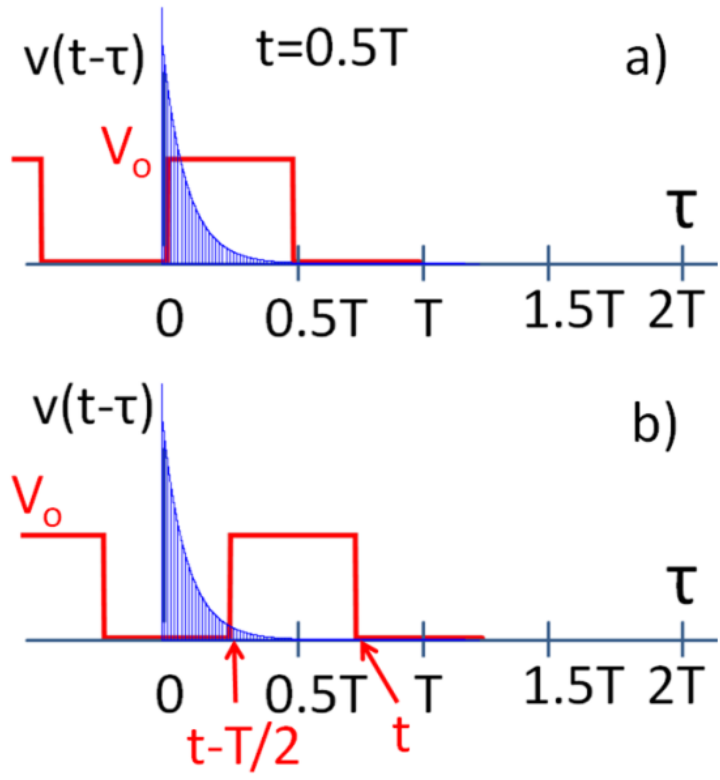

Figure 4. v(t- $\tau)$ vs $\tau$ for: a) $t=0.5 T$; and b) $0.5 T<t<T$

$$
v_{o}(t)=\int_{0.5 T}^{t} V_{0} \omega_{c} \exp \left(-\omega_{c} \tau\right) d \tau
$$

The same procedure can be applied at the other intervals that are shown in Figs. (5-7). The expected convolution integrals are shown by Eqs. (10-11).

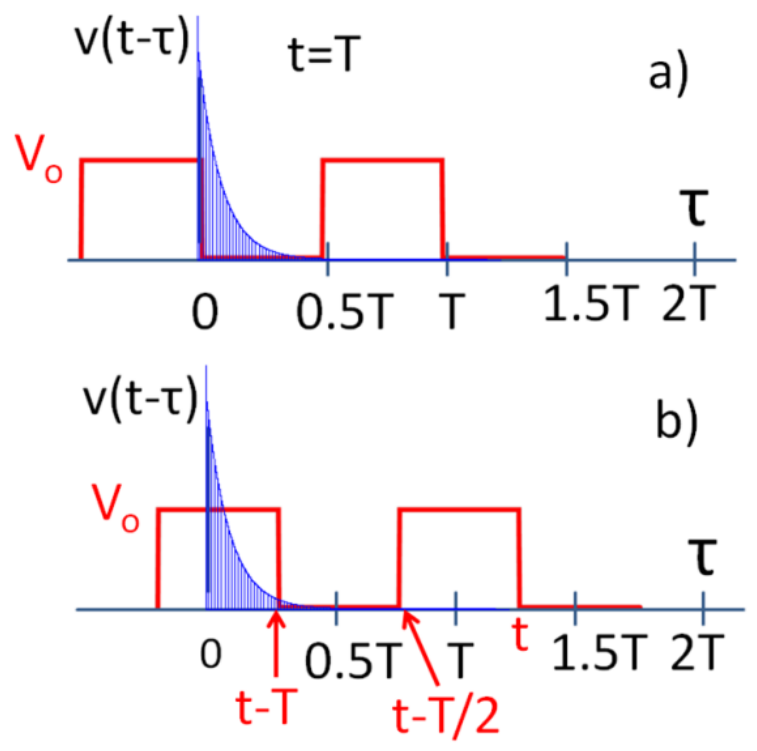

Figure 5. $v(t-\tau)$ vs $\tau$ for: a) $t=T$; and b) $T<t<1.5 T$ 


$$
\begin{aligned}
v_{o}(t)=\int_{0}^{t-T} V_{0} \omega_{c} \exp \left(-\omega_{c} \tau\right) d \tau \\
\\
\quad+\int_{t-0.5 T}^{t} V_{0} \omega_{c} \exp \left(-\omega_{c} \tau\right) d \tau
\end{aligned}
$$
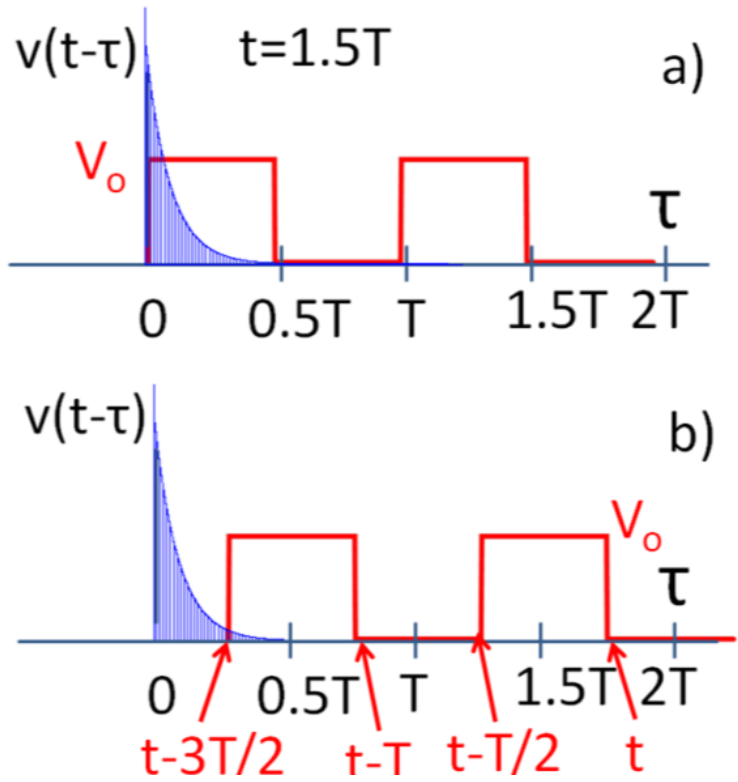

Figure 66. v(t- $\tau)$ vs $\tau$ for: a) $t=1.5 T$; and $b) 1.5 T<t<2 T$

$$
\begin{aligned}
v_{o}(t)=\int_{t-1.5 T}^{t-T} V_{0} \omega_{c} \exp \left(-\omega_{c} \tau\right) d \tau \\
+\int_{t-0.5 T}^{t} V_{0} \omega_{c} \exp \left(-\omega_{c} \tau\right) d \tau
\end{aligned}
$$

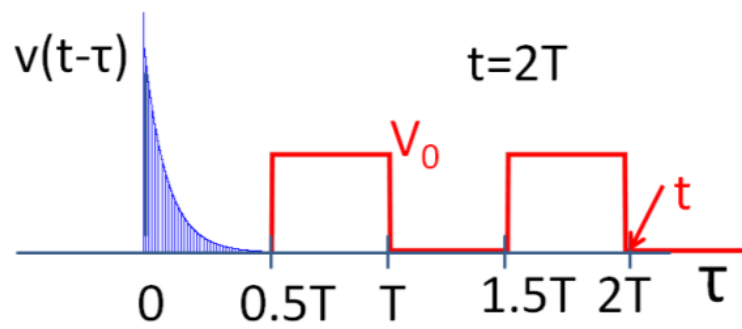

Figure 7. Plot of $v(t-\tau)$ vs $\tau$ for $t=2 T$

\section{The OUtPut Signals OF THE CONVOLUTION}

\section{INTEGRAL}

Depicted in Fig. 8(a) are the v vs. $t$ plots associated with the LPF/RC circuit shown in Fig. 1. The image was captured on an oscilloscope screen. The red line represents the input signal. The yellow line represents the output voltage, which is measured across the capacitor. Fig. 8(b) is a photograph of the corresponding experiment setup.
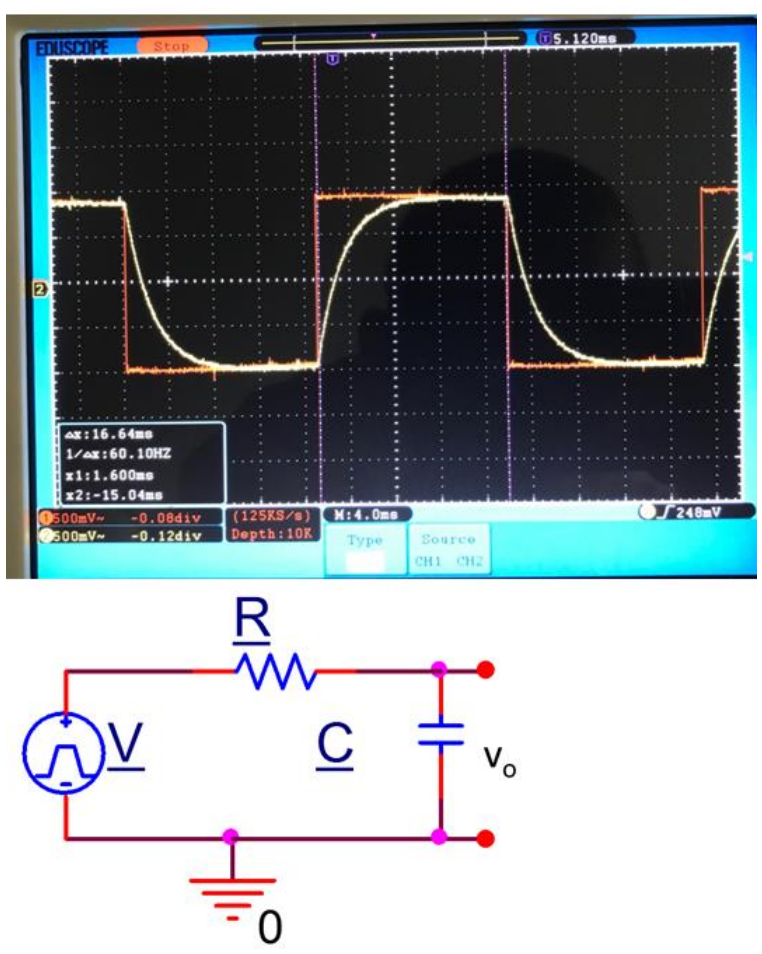

(a)

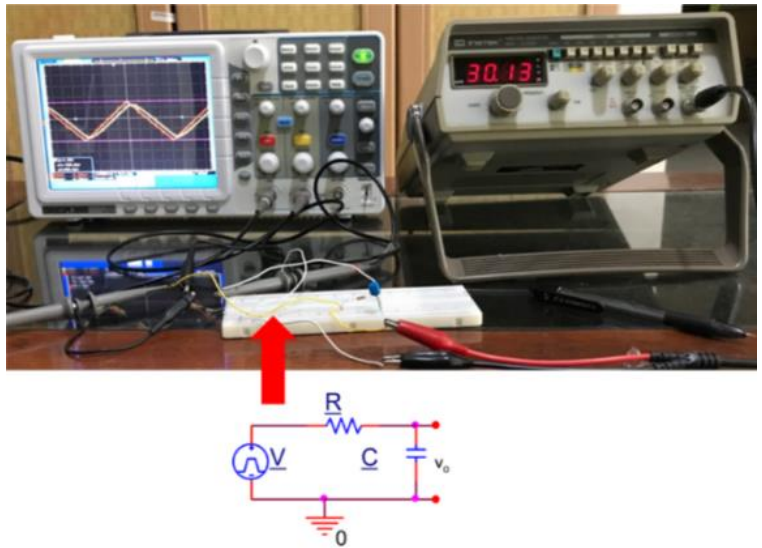

(b)

Figure 8. (a) The plot of $v$ vs. $t$ associated with the LPF/RC circuit where $R=2.2 \mathrm{k} \Omega, C=1.026 \mu \mathrm{F}$. The images were captured on an oscilloscope screen. The yellow and red lines are the output and input signals. The signal period is $33 \mathrm{~ms}$; (b) The $\mathrm{LPF} / \mathrm{RC}$ circuit experiment setup.

\section{THE GRAPHICAL TYPE OF SOLUTION}

The integral equations represented by Eqs. (8-11) and the corresponding graphs depicted in Figs (3-7) are based on positive voltage value, i.e., $0<\mathrm{v}<\mathrm{V}_{0}$. As a matter of choice and in order to mimic the experiment-based waveform associated with the square wave input signal in Fig. 8, from here and onward all plots are shifted downward by the amount of $0.5 \mathrm{~V}_{0}$ (see Figs. $(9-11,16)$ ). The lower panel in Fig. 9 depicts a simulated plot, which was generated by a superposition method by use of Eqs. (8-11). The PSpice simulation plot counterpart is shown in the upper panel. At $t=0^{+}$in Fig. 3(a), the impulse response and the input signal starts to convolve. As the leading edge 
of the input signal advances from 0 to $t$, the value of PROD continues to increase (Fig. 3(b)). The manifestation of this is shown in the output signal in Fig. 9 where it is increasing monotonically within the given time interval $(0<\tau<t)$ (see Fig 3(b)). As the input signal travels from $t$ (at the instant shown in Fig. 3(b)) towards $t=0.5 \mathrm{~T}$ the value of PROD does not change much and soon becomes constant. This fact is confirmed by the flat region of the output signal in Fig. 9 at this interval. As time continues to increase (the leading edge continue to advance) from $\mathrm{t}=0.5 \mathrm{~T}(16.5 \mathrm{~ms})$ to $\mathrm{t}=0.75 \mathrm{~T}(24.5 \mathrm{~ms})$ the overlapped regions start to decrease as shown in Figs. 4(a-b), which again is confirmed by the decrease in the output signal in Fig. 9. This cycle repeats at $\mathrm{t}=\mathrm{T}(33 \mathrm{~ms})$. Aided by Figs. (5-7), the foregoing reasoning is used to explain the shape of the output signal in Fig. 9 beyond T.

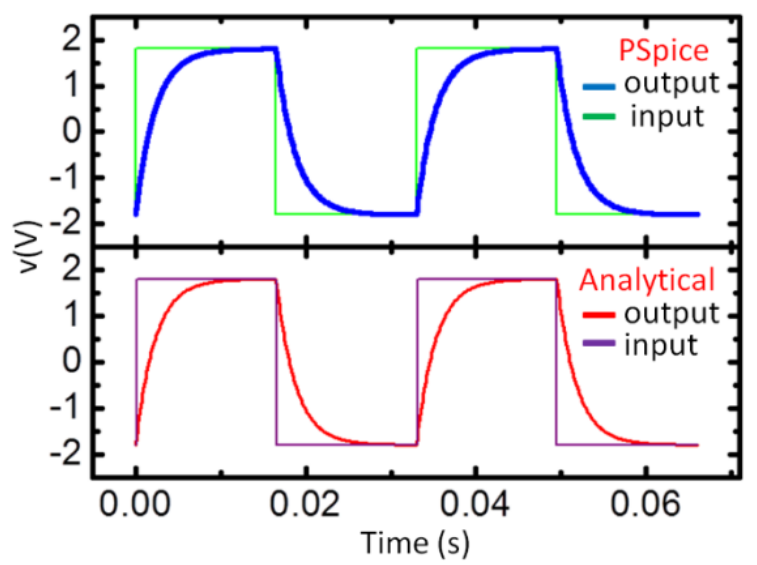

Figure 9. The plots of $v$ vs. $t$. $v$ representing the output voltage signal across the capacitor in Fig.1. Upper panel: PSpice based simulation result. Lower pane: convolution integral (analytical solution) based simulation, which was generated based on the superposition of Eqs. (8-11). $T=33 \mathrm{~ms}, R=2.2 \mathrm{k} \Omega$ and $C=1.026 \mu \mathrm{F}$.

Depicted in Fig. 10 is a composite v vs. $t$ plots associated with the analytical solution/convolution integral and experiment.

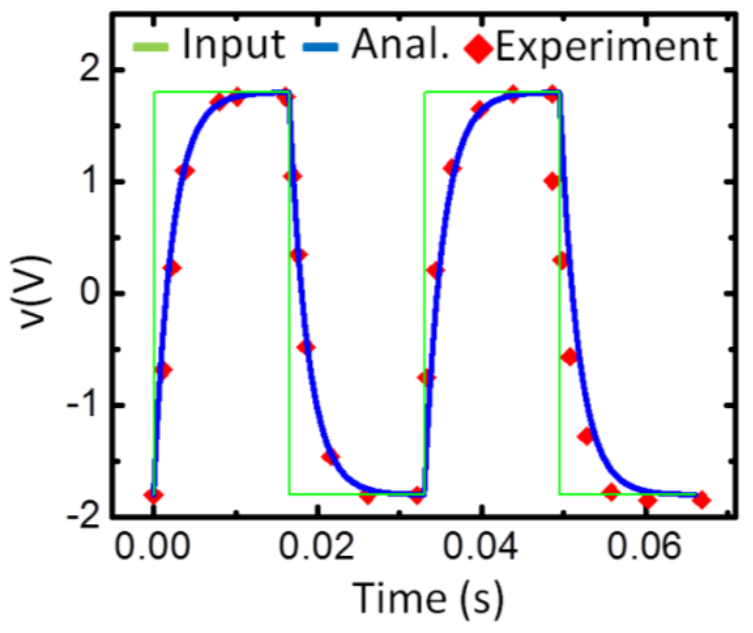

Figure 10. The plots of $v$ vs.t. representing experiment and analytical solution-based simulation. The impulse response is based on the $L P F / R C$ circuit in Fig. 1 , where $R=2.2 \mathrm{k} \Omega$ and $C=1.026 \mu F$. The green line represents an input signal. The blue line and scattered red diamonds represent the outputs.

The tasks of explaining: i) how Eqs. (8-11) have been utilized to generate the output signal in Fig. 9; and ii) how the respective graphical type of solutions associated with Figs. (3-7) can help explain the shape of the same output signal are completed. We now turn our attention to Fig. 11. Fig. 11 depicts a family of curves representing varying resistance values associated with the circuit schematic in Fig. 1. The plots in Fig. 11 were generated through the use of the analytical solution (Eqs. (8-11)). As shown, the lowest resistance value represents the blue signal. As the resistance increases the curvature of the plot increases as the plot itself gets shifted in the downward direction. The shifting can be explained in two different ways. The details are provided in the subsequent paragraphs.

First, at a fixed capacitance value $(\mathrm{C}=1.026 \mu \mathrm{F})$, the smaller the resistance value, the higher the cutoff frequency $\omega_{c}$ gets (see Eq. 4) leading to a higher decay rate of the impulse response. The upper and lower panels of Fig. 12 represent fast- and slow- exponentially decaying impulse response functions, respectively. The leading edges of the input signals (both in the upper and lower panels) in Fig 13 have passed the 0 s mark slightly. Notice that the region under the impulse response in the upper panel is slim but very tall leading to a larger value of PROD. On the other hand, the region under the impulse response in lower panel is wider but 13 times shorter resulting in a smaller PROD value. As before, the manifestation of these can be seen in Fig. 11, where the blue colored output signal representing $\mathrm{R}=500 \Omega$ has a higher value compared to that of the cyan colored output signal representing $\mathrm{R}=6.5 \mathrm{k} \Omega$. As the leading edges of the respective input signals advance to $\mathrm{t} \sim 15 \mathrm{~ms}$ in Fig. 14, the values of PROD associated with the upper and lower panels reach their respective maxima. This is confirmed by the blue $(\mathrm{R}=500 \Omega)$ and cyan $(\mathrm{R}=6.5 \mathrm{k} \Omega)$ output signals in Fig. 11 at $\mathrm{t} \sim 15 \mathrm{~ms}$. As the respective leading edges (see Fig. 15) reach $\mathrm{t} 24 \mathrm{~ms}$, the slim area impulse response in the upper panel is completely separated from the input signal, while the wider but shorter impulse response in the lower panel is still overlapping with the input signal. Fig. 11 confirms that at $\mathrm{t} \sim 24 \mathrm{~ms}$ the blue signal $(\mathrm{R}=500 \Omega)$ has a zero value but the cyan signal $(\mathrm{R}=6.5 \mathrm{k} \Omega)$ has a non-zero value.

Second, the respective time constants (the product of $\mathrm{R}$ and $\mathrm{C}$ ) associated with the $500-\Omega$ and $6.5-\mathrm{k} \Omega$ resistors are $0.51 \mathrm{~ms}$ and $6.7 \mathrm{~ms}$ suggesting that it takes longer for the $1.026-\mu \mathrm{F}$ capacitor to reach $63 \%$ of its maximum voltage (during charging) when the resistance $\mathrm{R}=6.5 \mathrm{k} \Omega$ is used. As shown in Fig. 11 , at $\mathrm{t}=6.7 \mathrm{~ms}$ the cyan signal $(6.5-\mathrm{k} \Omega)$ has reached only about $63 \%$ of the peak-to-peak voltage, while the blue signal representing the $500-\Omega$ resistor has attained the maximum voltage value at a much earlier time. At the time when the cyan signal $(6.5 \mathrm{k} \Omega)$ has arrived at $\mathrm{t}=16.5 \mathrm{~ms}$ its value starts to drop because the capacitor begins to discharge. Therefore, with the given the time constant $(6.7 \mathrm{~ms})$ and the input signal period $(\mathrm{T}=33 \mathrm{~ms})$, the cyan signal is not propagating fast enough. In turn, it will never reach the value of the input signal amplitude. The foregoing explanations apply to the green- and purplecolored signals in Fig. 11. 


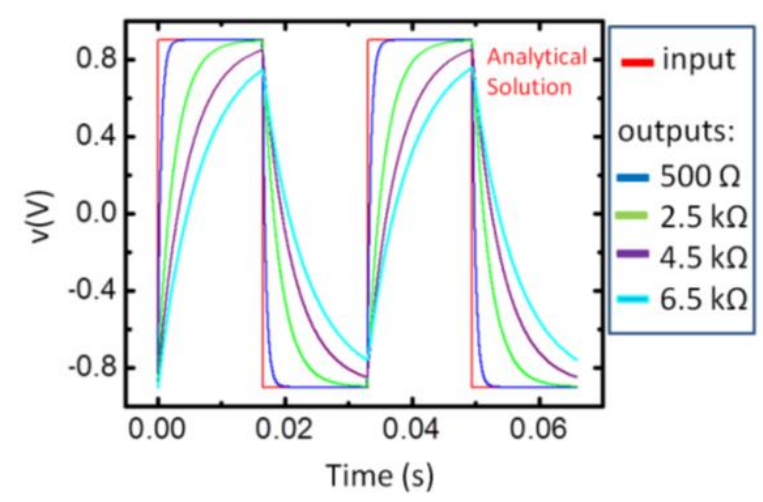

Figure 11. A square wave input. An analytical solution-based plots of $v$ vs. $t$ for varying $R$ values and fixed $C$ value. $C=1.026 \mu F$.

Red line: input signal. Curved lines: output signals. Refer to the legend.

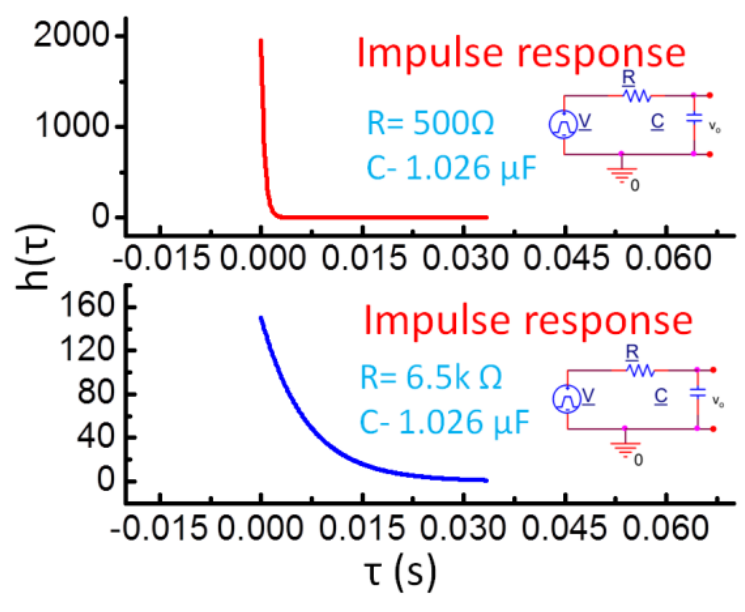

Figure 12. The plots $h(\tau)$ vs. $\tau$. Upper panel: $R=500 \Omega$ and $\mathrm{C}=1.026 \mu \mathrm{F}$ (fast exponentially decaying impulse response). Lower panel: $\mathrm{R}=6.5 \mathrm{k} \Omega$ (slower exponentially decaying impulse response).

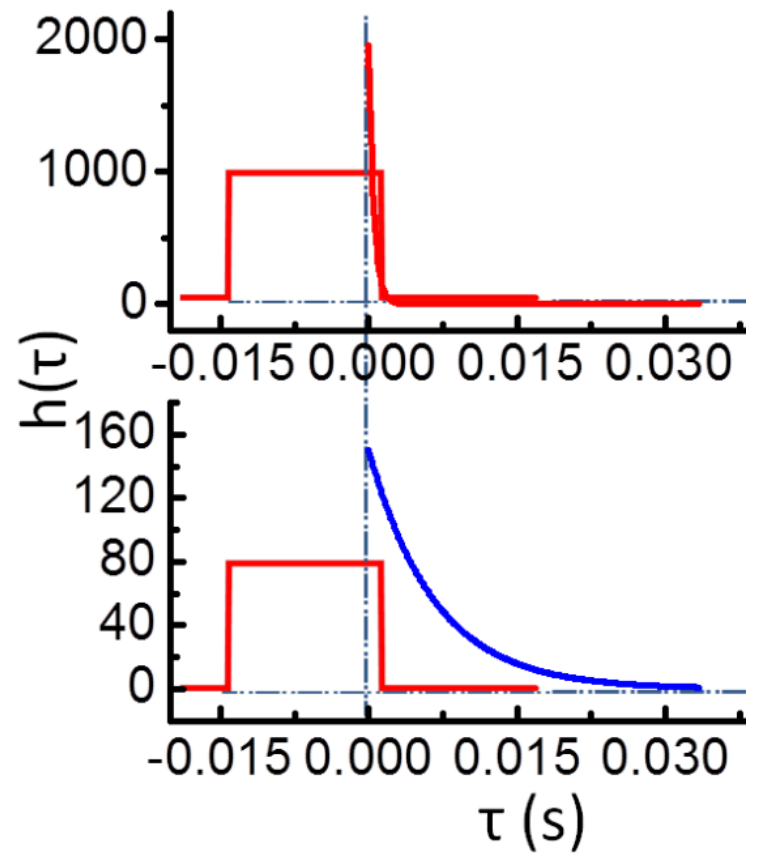

Figure 13. The convolution graphical type of solution. At the instant shown the leading edge of the input is located at $t$, which has passed $0 \mathrm{~s}$ slightly. The upper panel represents $R=500 \Omega$ and the lower one represents $R=6.5 \mathrm{k} \Omega$.

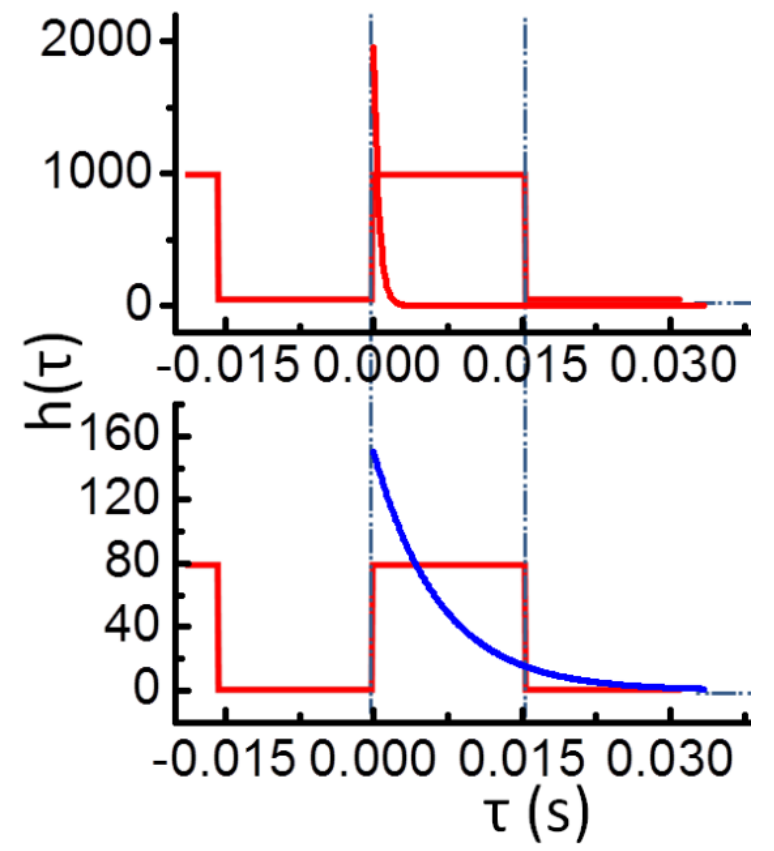

Figure 14 The convolution graphical type of solution. At the instant shown the value of $t$ is roughly $15 \mathrm{~ms}$. The upper panel represents $R=500 \Omega$ and the lower one represents $R=6.5 \mathrm{k} \Omega$.

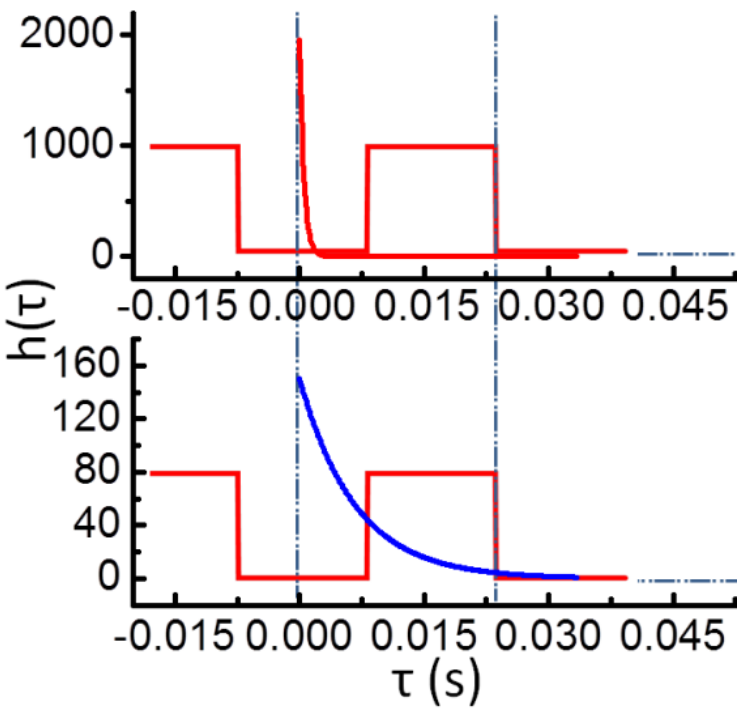

Figure 15 The convolution graphical type of solution. At the instant shown $t$ has passed $22.5 \mathrm{~ms}$ slightly. The upper panel represents $R=500 \Omega$ and the lower one represents $R=6.5 \mathrm{k} \Omega$.

Depicted in Fig. 16 are the plot of v vs. t for a varying capacitance values, while $\mathrm{R}$ value is held fixed at $2.2 \mathrm{k} \Omega$. The respective plots in upper and lower panels were generated through use of PSpice simulation and the analytical solution (Eqs. (8-11)). As shown the blue and orange plots in either panel are the representation of the 1$\mu \mathrm{F}$ and $11-\mu \mathrm{F}$ capacitance values, respectively. Each plot in Fig. 16 corresponds to a different time constant. 


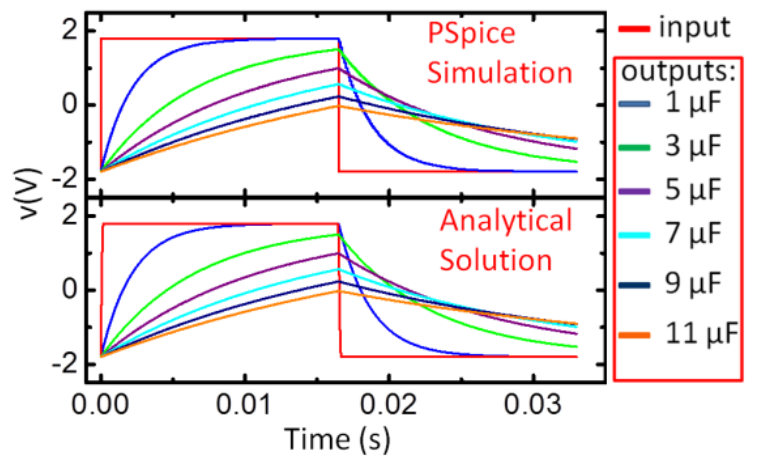

Figure 16. A square wave input. Plots of $v$ vs. $t$ for varying $C$ values and a fixed $R$ value. $R=2.2 \mathrm{k} \Omega$. $T=33 \mathrm{~ms}$. PSpice simulationbased plot (top) and analytical solution based (bottom).

\section{Conclusion}

A continuous time convolution (convolution integral) has been studied by incorporating a square wave as an input signal and an LPF/RC circuit acting as an impulse response. A semi graphical type of solutions was employed in order to attain the analytical solutions to the convolution integrals. That is to say that, graphs were used in order to conceptually determine the integral limits correctly. Finding the integral limits would be challenging in the present study as the input signal is not continuous, i.e., a square wave input signal. A full graphical type of solution was included allowing us to conceptually solve the convolution graphically. This was done by comparing the graphical type of solution and the corresponding analytical solution output signals. The role of time constant (the product of $\mathrm{R}$ and $\mathrm{C}$ ) in an $\mathrm{RC}$ circuit was emphasized allowing us to see the convolution from a Physics point of view rather than just seeing it as complicated and/or advanced mathematics. PSpice simulation and experiment results were included in order to motivate students to apply the convolution in a physical system such as the LPF/RC circuit.

\section{REFERENCE}

[1] B.P. Lathi and R. Green, "Time-domain Analysis of Continuoustime System," in Linear Systems and Signals, $3^{\text {rd }}$ ed. (Oxford, New York, NY 10016), Chap. 2, p. 180.

[2] I.S. Goldberg, M.G. Block and R.E. Rojas, "A Systematic Method for the Analytical Evaluation of Convolution Integrals," IEEE Transactions on Education (2002), vol. 45, pp. 65-69.

[3] M. Souare, V. Chankong, and C. Papachristou. "Undergraduate Notes on Convolutions and Integration by Parts," ASEE 2014 Zone Conference, University of Bridgeport, Bridgeport, CT, USA (2014), pp.??

[4] R. Nasr, S.R. Hall, and P. Garik, "Student Misconceptions in Signals and Systems and their Origins-Part II," $35^{\text {th }}$ ASEE/IEEE Frontiers in Education Conference, Indianapolis, IN, USA (2002), pp. TAE 26-31.

[5] J.K. Nelson and M.A. Hjalmarson, "Students' Understanding of Convolutions," IEEE International Conference on Acoustics, Speech and Signal Processing (ICASSP), Vancouver, BC, Canada (2013), pp. 4339-4343.

[6] R. Togneri and S. Male, "Signals and Systems: Casting It as an Action-Adventure Rather Than a Horror Genre," IEEE International Conference on Acoustics, Speech and Signal Processing (ICASSP), Brighton, UK (2019), pp. 7859-7863.

[7] A.V. Oppenheim, A.S. Willsky and S.H. Nawab "ContinuousTime LTI Systems: The Convolution Integral," in Signals and Systems, $2^{\text {nd }}$ ed. (Prentice Hall, New Jersey 07458, 1993), pp.90103.

[8] J.W. Nilsson and S.A. Riedel, "The Laplace Transform in Circuit Analysis," in Electric Circuits, 10th ed. (Pearson, New Jersey 07458, 2015), Chap. 13, pp. 487-493. 